\title{
Saturation number of Berge stars in random hypergraphs
}

\author{
Lele Liu* Changxiang He \\ College of Science \\ University of Shanghai for Science and Technology \\ Shanghai 200093, PR China \\ leliu@usst.edu.cn, changxiang-he@163.com \\ Liying $\mathrm{Kang}^{\dagger}$ \\ Department of Mathematics \\ Shanghai University \\ Shanghai 200444, PR China \\ lykang@shu.edu.cn
}

Submitted: Jan 19, 2020; Accepted: Nov 3, 2020; Published: Dec 11, 2020

(C) The authors. Released under the CC BY-ND license (International 4.0).

\begin{abstract}
Let $G$ be a graph. We say an $r$-uniform hypergraph $H$ is a Berge- $G$ if there exists a bijection $\phi: E(G) \rightarrow E(H)$ such that $e \subseteq \phi(e)$ for each $e \in E(G)$. Given a family of $r$-uniform hypergraphs $\mathcal{F}$ and an $r$-uniform hypergraph $H$, a spanning sub-hypergraph $H^{\prime}$ of $H$ is $\mathcal{F}$-saturated in $H$ if $H^{\prime}$ is $\mathcal{F}$-free, but adding any edge in $E(H) \backslash E\left(H^{\prime}\right)$ to $H^{\prime}$ creates a copy of some $F \in \mathcal{F}$. The saturation number of $\mathcal{F}$ is the minimum number of edges in an $\mathcal{F}$-saturated spanning sub-hypergraph of $H$. In this paper, we asymptotically determine the saturation number of Berge stars in random $r$-uniform hypergraphs.
\end{abstract}

Mathematics Subject Classifications: 05C65, 05C35, 05C80

\section{Introduction}

Given a family of graphs $\mathcal{F}$, a graph $G$ is $\mathcal{F}$-saturated if $G$ does not contain any $F \in \mathcal{F}$ as a subgraph, but adding any missing edge to $G$ creates a copy of some $F \in \mathcal{F}$. In other

\footnotetext{
*Supported by the National Nature Science Foundation of China (No. 12001370)

${ }^{\dagger}$ Corresponding author. Supported by the National Nature Science Foundation of China (Nos. $11871329,11971298)$
} 
words, $G$ is $\mathcal{F}$-saturated if and only if it is an edge-maximal $\mathcal{F}$-free graph. The maximum possible number of edges in a graph $G$ that is $\mathcal{F}$-saturated is known as the Turán number of $\mathcal{F}$. The study of Turán numbers for various families of graphs is a cornerstone of extremal combinatorics.

On the other hand, the minimum number of edges in an $\mathcal{F}$-saturated graph with $n$ vertices, denoted by $\operatorname{sat}(n, \mathcal{F})$, is called the saturation number of $\mathcal{F}$. Saturation numbers were first studied by Erdös, Hajnal and Moon [6] and since then have been researched extensively. In 1986, Kászonyi and Tuza [9] showed that saturation numbers are always linear. That is, $\operatorname{sat}(n, F)=O(n)$ for any graph $F$. In the same paper, they also determined the saturation number of star $K_{1, s}$. To be specific, they proved that

$$
\operatorname{sat}\left(n, K_{1, s}\right)= \begin{cases}\left(\begin{array}{c}
n-s \\
2
\end{array}\right)+\left(\begin{array}{c}
s \\
2
\end{array}\right), & \text { if } s+1 \leqslant n<3 s / 2, \\
\left\lceil\frac{(s-1) n}{2}-\frac{s^{2}}{8}\right\rceil, & \text { if } n \geqslant 3 s / 2 .\end{cases}
$$

For more results on graph saturation, we refer the reader to the survey [7].

Graph saturation has been generalized in several natural ways, including studying other host graphs besides the complete graph, and the saturation number of hypergraphs. Recall that a hypergraph $H=(V(H), E(H))$ is a pair consisting of a vertex set $V(H)$, and a set $E(H)$ of subsets of $V(H)$, the edges of $H$. An $r$-uniform hypergraph or simply $r$-graph is a hypergraph such that all its edges have size $r$. Throughout this paper, we always assume that $r \geqslant 2$ is an integer.

To state our result precisely, we introduce some terminology and notation. Given a family of $r$-uniform hypergraphs $\mathcal{F}$ and an $r$-uniform hypergraph $H$, a spanning subhypergraph $H^{\prime}$ of $H$ is $\mathcal{F}$-saturated in $H$ if $H^{\prime}$ is $\mathcal{F}$-free, but adding any edge in $E(H) \backslash E\left(H^{\prime}\right)$ to $H^{\prime}$ creates a copy of some $F \in \mathcal{F}$. The minimum number of edges in an $\mathcal{F}$-saturated spanning sub-hypergraph of $H$ is called the saturation number of $\mathcal{F}$, denoted by $\operatorname{sat}(H, \mathcal{F})$. Note that with this general notation, $\operatorname{sat}(n, \mathcal{F})=\operatorname{sat}\left(K_{n}^{(r)}, \mathcal{F}\right)$, where $K_{n}^{(r)}$ is the complete $r$-uniform hypergraph on $n$ vertices.

Let $G$ be a graph. We say an $r$-uniform hypergraph $H$ is a Berge- $G$ if there exists a bijection $\phi: E(G) \rightarrow E(H)$ such that $e \subseteq \phi(e)$ for each edge $e \in E(G)$. Recently, extremal problems for Berge hypergraphs have attracted the attention of a lot of researchers, see, e.g., [3, 2, 4, 5, 8, 13]. In 2018, Austhof and English [2] studied the saturation number of Berge stars. They proved that

$$
\operatorname{sat}\left(n, \text { Berge }-K_{1, s}\right)=\min _{a \in[n],\left(\begin{array}{c}
a-1 \\
r-1
\end{array}\right) \leqslant s-2}\left\lceil\frac{(s-1)(n-a)}{r}\right\rceil+\left(\begin{array}{l}
a \\
r
\end{array}\right)
$$

for large $n$, which generalizes equation (1) to uniform hypergraphs. In 2019, English et al. [5] proved that sat $(n, B$ erge- $F)=O(n)$ for any graph $F$ and uniformities $3 \leqslant r \leqslant 5$.

In recent years, some classic extremal problems were extended to random analogues. The random $r$-uniform hypergraph $H^{r}(n, p)$ is the probability space of all $r$-uniform hypergraphs with vertex set $[n]:=\{1,2, \ldots, n\}$, and each edge is chosen with probability 
$p$ independently of all the other edges. In particular, for $r=2$ this model reduces to the well known Erdős-Rényi random graph $G(n, p)$. In 2017, Korándi and Sudakov [10] initiated the study of graph saturation in random graphs. More precisely, they proved that with high probability

$$
\operatorname{sat}\left(G(n, p), K_{m}\right)=(1+o(1)) n \cdot \log _{1 /(1-p)} n
$$

for each fixed $p \in(0,1)$. Let us recall that an event holds with high probability (w.h.p. for short) in $G(n, p)$ if its probability goes to 1 as $n$ tends to infinity. In 2018, Mohammadian and Tayfeh-Rezaie [11] asymptotically determined the saturation number of stars in random graphs. It is proved that w.h.p.

$$
\operatorname{sat}\left(G(n, p), K_{1, s}\right)=\frac{(s-1) n}{2}-(1+o(1))(s-1) \cdot \log _{1 /(1-p)} n
$$

for every fixed $p \in(0,1)$, which supplements the early work of Zito [14].

The main goal of this paper is to extend (2) to random hypergraphs. To be specific, we asymptotically determine the saturation number of Berge stars in random $r$-uniform hypergraphs.

Using a similar but more complicated technique than that of [11], we can prove the main result of this paper.

Theorem 1. Let $p \in(0,1)$ be a fixed number and $s \geqslant 2$. Then w.h.p.

$$
\operatorname{sat}\left(H^{r}(n, p), \text { Berge }-K_{1, s}\right)=\frac{s-1}{r}\left(n-(1+o(1))\left(r ! \cdot \log _{1 /(1-p)} n\right)^{1 /(r-1)}\right) .
$$

Note that the proof of Theorem 1 is a combination of Theorem 4 and Theorem 9 . Taking $r=2$, we obtain the result of Mohammadian and Tayfeh-Rezaie described in Equation (2), so Theorem 1 generalizes that result. Let us note that most of our results are about $n$ tending to infinity, so we tacitly assume that $n$ is large enough throughout this paper.

\section{Lower bound on the saturation number of Berge stars}

We start this section with some notation. Let $H$ be a hypergraph and $S$ be a subset of $V(H)$, the sub-hypergraph of $H$ induced by $S$ is the hypergraph $H[S]$ consisting of all edges of $H$ that are contained in $S$. A set $X \subseteq V(H)$ is an independent set of $H$ if $X$ contains no edges of $H$. We also denote by $\alpha_{k}(H)$ the maximum cardinality of a subset $S$ of $V(H)$ such that the maximum degree of $H[S]$ is at most $k$.

Lemma 2 ([11]). Let $X$ be a binomial random variable with parameters $n$ and $p \in(0,1)$. Then

$$
\mathbf{P}(X \leqslant s) \leqslant\left(\begin{array}{l}
n \\
s
\end{array}\right)(1-p)^{n-s}
$$

for any $s \in\{0,1, \ldots, n\}$. 
Lemma 3. Let $p \in(0,1)$ be a fixed number and $k \geqslant 1$. Then w.h.p.

$$
\alpha_{k}\left(H^{r}(n, p)\right) \leqslant(1+o(1))\left(r ! \cdot \log _{1 /(1-p)} n\right)^{1 /(r-1)} .
$$

Proof. For convenience, we denote $q:=1 /(1-p)$ and

$$
i:=\left\lceil\left(r ! \cdot \log _{q} n+k(r-1) ! \cdot \log _{q} \log _{q} n\right)^{1 /(r-1)}\right\rceil+r-1 .
$$

Let $X_{i}$ be the number of induced sub-hypergraphs in $H^{r}(n, p)$ on $i$ vertices with at most $\lfloor i k / r\rfloor$ edges. For any $A \subseteq V\left(H^{r}(n, p)\right)$ with $|A|=i$, let $Y_{A}$ be the number of edges in $H^{r}(n, p)[A]$.

Our first goal is to estimate the expectation $\mathbf{E}\left(X_{i}\right)$. By Theorem 2 we have

$$
\mathbf{P}\left(Y_{A} \leqslant\lfloor i k / r\rfloor\right) \leqslant\left(\begin{array}{c}
\left(\begin{array}{c}
i \\
r
\end{array}\right) \\
\lfloor i k / r\rfloor
\end{array}\right)(1-p)^{\left(\begin{array}{l}
i \\
r
\end{array}\right)-\lfloor i k / r\rfloor} .
$$

Noting that the function

$$
f(x):=\left(\frac{e\left(\begin{array}{c}
i \\
r
\end{array}\right)}{x}\right)^{x}
$$

is non-decreasing in $x \in\left(0,\left(\begin{array}{l}i \\ r\end{array}\right)\right)$, we have

$$
\begin{aligned}
\mathbf{P}\left(Y_{A} \leqslant\lfloor i k / r\rfloor\right) & \leqslant\left(\frac{e\left(\begin{array}{c}
i \\
r
\end{array}\right)}{\lfloor i k / r\rfloor}\right)^{\lfloor i k / r\rfloor}(1-p)^{\left(\begin{array}{c}
i \\
r
\end{array}\right)-\lfloor i k / r\rfloor} \\
& \leqslant\left(\frac{r e\left(\begin{array}{l}
i \\
r
\end{array}\right)}{i k}\right)^{i k / r}(1-p)^{\left(\begin{array}{c}
i \\
r
\end{array}\right)-i k / r} .
\end{aligned}
$$

In view of the above inequality, we can give an estimation of $\mathbf{E}\left(X_{i}\right)$ as follows:

$$
\begin{aligned}
\mathbf{E}\left(X_{i}\right) & =\sum_{A \subseteq V\left(H^{r}(n, p)\right),|A|=i} \mathbf{P}\left(Y_{A} \leqslant\lfloor i k / r\rfloor\right) \\
& \leqslant\left(\begin{array}{c}
n \\
i
\end{array}\right)\left(\frac{r e\left(\begin{array}{l}
i \\
r
\end{array}\right)}{i k}\right)^{i k / r}(1-p)^{\left(\begin{array}{c}
i \\
r
\end{array}\right)-i k / r} \\
& \leqslant\left(\frac{n e}{i}\right)^{i}\left(\frac{r e\left(\begin{array}{c}
i \\
r
\end{array}\right)}{i k}\right)^{i k / r}(1-p)^{\left(\begin{array}{l}
i \\
r
\end{array}\right)-i k / r} \\
& \leqslant\left(\left(\frac{e^{r+1+r / k}}{(1-p) r^{r-1} k}\right)^{k} i^{(r-1) k-r} n^{r}(1-p)^{\left(\begin{array}{c}
i-1 \\
r-1
\end{array}\right)}\right)^{i / r} .
\end{aligned}
$$


To finish the proof, we shall prove that $i^{(r-1) k-r} n^{r}(1-p)^{\left(\begin{array}{c}i-1 \\ r-1\end{array}\right)}=o(1)$, and therefore $\mathbf{E}\left(X_{i}\right)=o(1)$. Indeed, by some algebra we have

$$
\begin{aligned}
i^{(r-1) k-r} n^{r}(1-p)^{\left(\begin{array}{c}
i-1 \\
r-1
\end{array}\right)} & \leqslant\left(2\left(r ! \cdot \log _{q} n\right)^{1 /(r-1)}\right)^{(r-1) k-r} n^{r}(1-p)^{\left(\begin{array}{c}
i-1 \\
r-1
\end{array}\right)} \\
& \leqslant\left(2^{r-1} r !\right)^{k}\left(\log _{q} n\right)^{k-1} n^{r}(1-p)^{(i-r+1)^{r-1} /(r-1) !} \\
& \leqslant \frac{\left(2^{r-1} r !\right)^{k}}{\log _{q} n}
\end{aligned}
$$

the last inequality follows from the fact that $(1-p)^{(i-r+1)^{r-1} /(r-1) !} \leqslant n^{-r}\left(\log _{q} n\right)^{-k}$ by $(3)$. Therefore, $\mathbf{E}\left(X_{i}\right)=o(1)$.

Finally, by the Markov inequality, $\mathbf{P}\left(X_{i}>0\right) \leqslant \mathbf{E}\left(X_{i}\right)=o(1)$. Hence, w.h.p. $X_{i}=$ 0 , which yields that for any $X \subseteq V\left(H^{r}(n, p)\right)$ with $|X|=i$, the number of edges of $H^{r}(n, p)[X]$ is at least $\lfloor i k / r\rfloor+1$. Hence, the maximum degree $\Delta$ of $H^{r}(n, p)[X]$ satisfies $i \Delta \geqslant r(\lfloor i k / r\rfloor+1)$. Consequently,

$$
\Delta \geqslant \frac{r(\lfloor i k / r\rfloor+1)}{i}>k
$$

Therefore, we have

$$
\alpha_{k}\left(H^{r}(n, p)\right)<i=(1+o(1))\left(r ! \cdot \log _{1 /(1-p)} n\right)^{1 /(r-1)},
$$

completing the proof of Theorem 3.

In view of Theorem 3, we can obtain a lower bound of the saturation number of Berge stars.

Theorem 4. Let $p \in(0,1)$ be a fixed number and $s \geqslant 2$. Then w.h.p.

$$
\operatorname{sat}\left(H^{r}(n, p), \operatorname{Berge}-K_{1, s}\right) \geqslant \frac{s-1}{r}\left(n-(1+o(1))\left(r ! \cdot \log _{1 /(1-p)} n\right)^{1 /(r-1)}\right) .
$$

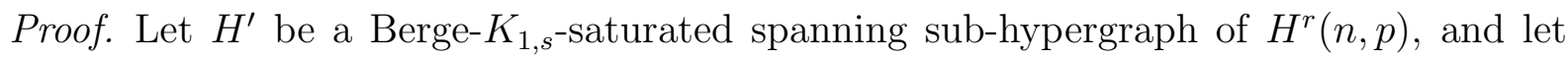
$A \subseteq V\left(H^{\prime}\right)$ be the set of vertices with degree at most $s-2$ in $H^{\prime}$. Then each vertex in $V\left(H^{r}(n, p)\right) \backslash A$ is of degree at least $s-1$ in $H^{\prime}$. Therefore,

$$
r\left|E\left(H^{\prime}\right)\right| \geqslant \sum_{v \in V\left(H^{r}(n, p)\right) \backslash A} \operatorname{deg}_{H^{\prime}}(v) \geqslant(s-1)(n-|A|) .
$$

Clearly, $H^{\prime}[A]=H^{r}(n, p)[A]$. Hence, $|A| \leqslant \alpha_{s-2}\left(H^{r}(n, p)\right)$. It follows from Theorem 3 that

$$
\begin{aligned}
\left|E\left(H^{\prime}\right)\right| & \geqslant \frac{(s-1)(n-|A|)}{r} \\
& \geqslant \frac{s-1}{r}\left(n-(1+o(1))\left(r ! \cdot \log _{1 /(1-p)} n\right)^{1 /(r-1)}\right),
\end{aligned}
$$

completing the proof of Theorem 4 . 


\section{Upper bound on the saturation number of Berge stars}

In this section, we will give an upper bound on the saturation number of Berge stars in random hypergraphs. Before continuing, we need the following lemma.

Lemma 5 ([12]). Let $H$ be a fixed $r$-uniform hypergraph on $n$ vertices with maximum degree $\Delta$. There exists a constant $C>0$ such that for $p=C(\ln n / n)^{1 / \Delta}$, w.h.p. the random r-uniform hypergraph $H^{r}(n, p)$ contains a copy of $H$.

Lemma 6. Let $r \geqslant 2$ and $a=\left\lfloor\left(r ! \cdot\left(\log _{1 /(1-p)} n-3 \cdot \log _{1 /(1-p)} \log _{1 /(1-p)} n\right)\right)^{1 /(r-1)}\right\rfloor-2$, and

$$
A_{i}=\frac{\left(\begin{array}{c}
a \\
i
\end{array}\right)\left(\begin{array}{c}
n-a \\
a-i
\end{array}\right)}{\left(\begin{array}{c}
n \\
a
\end{array}\right)(1-p)^{\left(\begin{array}{c}
i \\
r
\end{array}\right)}}, i \geqslant r
$$

Then $\lim _{n \rightarrow \infty} \sum_{i=r}^{a} A_{i}=0$.

Proof. For any $r \leqslant i \leqslant a$, our main goal is to show that $A_{i} \leqslant A_{r}$. To this end, note that $A_{r+1} \leqslant A_{r}$, hence it suffices to show that $A_{i} \leqslant A_{r}$ for $i \geqslant r+2$.

By simple algebra, we get

$$
\begin{aligned}
\frac{A_{j+1}}{A_{j}} & =\frac{(a-j)^{2}}{(j+1)(n-2 a+j+1)} \cdot(1-p)^{\left(\begin{array}{c}
j \\
r
\end{array}\right)-\left(\begin{array}{c}
j+1 \\
r
\end{array}\right)} \\
& \leqslant\left(1+O\left(\frac{\left(\log _{1 /(1-p)} n\right)^{1 /(r-1)}}{n}\right)\right) \frac{a^{2}(1-p)^{\left(\begin{array}{c}
j \\
r
\end{array}\right)-\left(\begin{array}{c}
j+1 \\
r
\end{array}\right)}}{(j+1) n} .
\end{aligned}
$$

Therefore, we obtain

$$
\frac{A_{i}}{A_{r}}=\prod_{j=r}^{i-1} \frac{A_{j+1}}{A_{j}} \leqslant(1+o(1))\left(\frac{a^{2}}{n}\right)^{i-r} \frac{r !(1-p)^{1-\left(\begin{array}{c}
i \\
r
\end{array}\right)}}{i !} .
$$

Noting that $e>(1+1 / j)^{j}$ for each $j \in[i]$, we have $i !>((i+1) / e)^{i}>(i / e)^{i}$. Therefore,

$$
\frac{A_{i}}{A_{r}} \leqslant r !(1+o(1))\left(\frac{a^{2}}{n}\right)^{i-r}\left(\frac{e}{i}\right)^{i}(1-p)^{1-\left(\begin{array}{c}
i \\
r
\end{array}\right) .}
$$

To simplify inequality (4), we need the following claim.

Claim 7. $r !\left(\begin{array}{l}i \\ r\end{array}\right) \leqslant(i-r)(i+2)^{r-1}$, where $i \geqslant r+2$.

Proof of Theorem $\%$. Assume that $i \geqslant r+2$, then we have

$$
\begin{aligned}
r !\left(\begin{array}{c}
i \\
r
\end{array}\right) & =\frac{i !}{(i-r+2) !} \cdot(i-r+2)(i-r+1) \\
& \leqslant(i+2)^{r-2}(i-r+2)(i-r+1) \\
& \leqslant(i-r)(i+2)^{r-1} .
\end{aligned}
$$


The last inequality follows from a simple fact that

$$
(i-r)(i+2)-(i-r+2)(i-r+1)=(r-1)(i-r)-2 \geqslant 0 .
$$

The proof of the claim is completed.

Finally, in view of (4) and Theorem 7 we deduce that

$$
\begin{aligned}
\frac{A_{i}}{A_{r}} & \leqslant r !(1-p)(1+o(1))\left(\frac{a^{2} e}{n i}(1-p)^{-\left(\begin{array}{l}
i \\
r
\end{array}\right) /(i-r)}\right)^{i-r} \\
& \leqslant r !(1-p)(1+o(1))\left(\frac{a^{2} e}{n i}(1-p)^{-(i+2)^{r-1} / r !}\right)^{i-r} .
\end{aligned}
$$

Since $i \leqslant a$, we have

$$
(1-p)^{-(i+2)^{r-1} / r !} \leqslant \frac{n}{\left(\log _{1 /(1-p)} n\right)^{3}} .
$$

Combining these two inequalities, we see

$$
\frac{A_{i}}{A_{r}} \leqslant r !(1-p)(1+o(1))\left(\frac{a^{2} e}{i\left(\log _{1 /(1-p)} n\right)^{3}}\right)^{i-r} \leqslant 1 .
$$

It follows that

$$
\sum_{i=r}^{a} A_{i} \leqslant a A_{r}=\frac{a\left(\begin{array}{c}
a \\
r
\end{array}\right)\left(\begin{array}{c}
n-a \\
a-r
\end{array}\right)}{(1-p)\left(\begin{array}{l}
n \\
a
\end{array}\right)}=o(1),
$$

completing the proof of Theorem 6 .

An $r$-uniform hypergraph is nearly-d-regular if every vertex has degree either $d$ or $d-1$, and less than $r$ vertices have degree $d-1$. A hypergraph $H$ is called linear if every pair of edges intersects in at most one vertex. The following theorem guarantees the existence of nearly-regular linear hypergraphs.

Theorem 8 ([2]). Let $d \geqslant 1$ and $r \geqslant 2$. Then for all sufficiently large $n$, there exists a nearly-d-regular $r$-uniform linear hypergraph on $n$ vertices.

Armed with Theorem 5, Theorem 6 and Theorem 8, we are ready to propose our main result in this section.

Theorem 9. Let $p \in(0,1)$ be a fixed number. Then w.h.p.

$$
\operatorname{sat}\left(H^{r}(n, p), \operatorname{Berge}-K_{1, s}\right) \leqslant \frac{s-1}{r}\left(n-(1+o(1))\left(r ! \cdot \log _{1 /(1-p)} n\right)^{1 /(r-1)}\right) .
$$

Proof. For short, we denote $H:=H^{r}(n, p)$ and let

$$
a=\left\lfloor\left(r ! \cdot\left(\log _{1 /(1-p)} n-3 \cdot \log _{1 /(1-p)} \log _{1 /(1-p)} n\right)\right)^{1 /(r-1)}\right\rfloor-2 .
$$


Fix a nearly- $(s-1)$-regular $r$-uniform linear hypergraph $R$ on $n-a$ vertices. For any $A \subseteq V(H)$ with $|A|=a$, let

$$
X_{A}= \begin{cases}1, & \text { if } A \text { is an independent set in } H \text { and } R \subseteq H[V(H) \backslash A], \\ 0, & \text { otherwise, }\end{cases}
$$

and set $X:=\sum_{A \subseteq V(H),|A|=a} X_{A}$.

Our first goal is to show that $\mathbf{P}(X=0)=o(1)$ with high probability. To this end, note that for any $0<\varepsilon<1 / 5$ we have

$$
\mathbf{E}\left(X_{A}\right) \geqslant(1-p)^{\left(\begin{array}{l}
a \\
r
\end{array}\right)}(1-\varepsilon)
$$

from Theorem 5. By linearity of expectation we deduce that

$$
\mathbf{E}(X) \geqslant\left(\begin{array}{l}
n \\
a
\end{array}\right)(1-p)^{\left(\begin{array}{c}
a \\
r
\end{array}\right)}(1-\varepsilon) .
$$

Moreover, for subsets $S, T \subseteq V(H)$ of size $a$ with $|S \cap T|=i$, we find that

$$
\mathbf{E}\left(X_{S} X_{T}\right) \leqslant(1-p)^{2\left(\begin{array}{l}
a \\
r
\end{array}\right)-\left(\begin{array}{l}
i \\
r
\end{array}\right)},
$$

where $\left(\begin{array}{l}i \\ r\end{array}\right)=0$ if $i \leqslant r-1$.

By the Chebyshev's inequality (See [1, Theorem 4.3.1]), we have

$$
\mathbf{P}(X=0) \leqslant \frac{\operatorname{Var}(X)}{\mathbf{E}(X)^{2}}=\sum_{\substack{S, T \subseteq V(H),|S|=|T|=a}} \frac{\mathbf{E}\left(X_{S} X_{T}\right)-\mathbf{E}\left(X_{S}\right) \cdot \mathbf{E}\left(X_{T}\right)}{\mathbf{E}(X)^{2}} .
$$

It follows from $(5)-(7)$ and Theorem 6 that

$$
\begin{aligned}
& \mathbf{P}(X=0) \leqslant \sum_{i=0}^{a} \sum_{\substack{S, T \subseteq V(H),|S|=|T|=a,|S \cap T|=i}} \frac{\mathbf{E}\left(X_{S} X_{T}\right)-\mathbf{E}\left(X_{S}\right) \cdot \mathbf{E}\left(X_{T}\right)}{\mathbf{E}(X)^{2}}
\end{aligned}
$$

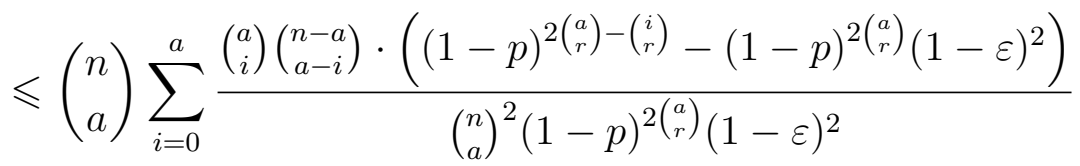

$$
\begin{aligned}
& =\sum_{i=0}^{a} \frac{\left(\begin{array}{c}
a \\
i
\end{array}\right)\left(\begin{array}{c}
n-a \\
a-i
\end{array}\right)}{\left(\begin{array}{l}
n \\
a
\end{array}\right)(1-p)^{\left(\begin{array}{c}
i \\
r
\end{array}\right)}} \cdot \frac{1-(1-p)^{\left(\begin{array}{c}
i \\
r
\end{array}\right)}(1-\varepsilon)^{2}}{(1-\varepsilon)^{2}} \\
& \leqslant 3 \varepsilon \sum_{i=0}^{r-1} \frac{\left(\begin{array}{c}
a \\
i
\end{array}\right)\left(\begin{array}{c}
n-a \\
a-i
\end{array}\right)}{\left(\begin{array}{l}
n \\
a
\end{array}\right)}+2 \sum_{i=r}^{a} \frac{\left(\begin{array}{c}
a \\
i
\end{array}\right)\left(\begin{array}{c}
n-a \\
a-i
\end{array}\right)}{\left.\left(\begin{array}{c}
n \\
a
\end{array}\right)(1-p)^{(i} r\right)} \\
& =o(1) \text {. }
\end{aligned}
$$

Hence, $\mathbf{P}(X=0)=o(1)$. This shows that w.h.p. there is $A \subseteq V(H)$ with $|A|=a$ such that $A$ is an independent set in $H$ and $H[V(H) \backslash A]$ has a copy of $R$ as a sub-hypergraph. 
Let $H^{\prime}$ be a Berge- $K_{1, s^{-}}$saturated spanning sub-hypergraph of $H$ such that

$$
H^{\prime}[V(H) \backslash A]=R
$$

Denote by $\alpha$ and $\beta$ the numbers of vertices of degree $s-1$ and $s-2$ in $H^{\prime}[V(H) \backslash A]$ respectively, where $\alpha+\beta=n-a$ and $\beta \leqslant r-1$. Clearly, the number of edges in $H^{\prime}$ which have nonempty intersection with $A$ and $V(H) \backslash A$ is at most $\beta$. Therefore,

$$
\begin{aligned}
r\left|E\left(H^{\prime}\right)\right| & \leqslant \alpha(s-1)+\beta(s-2)+r \beta \\
& =(s-1)(\alpha+\beta)+(r-1) \beta \\
& \leqslant(s-1)(n-a)+(r-1)^{2},
\end{aligned}
$$

which yields that

$$
\left|E\left(H^{\prime}\right)\right| \leqslant \frac{s-1}{r}\left(n-(1+o(1))\left(r ! \cdot \log _{1 /(1-p)} n\right)^{1 /(r-1)}\right)
$$

completing the proof.

\section{Acknowledgements}

The authors would like to thank the anonymous referees for valuable suggestions, which have considerably improved the presentation of the paper.

\section{References}

[1] N. Alon, J. Spencer. The Probabilistic Method, 4th Edition, John Wiley \& Sons, 2016.

[2] B. Austhof, S. English. Nearly-regular hypergraphs and saturation of Berge stars. Electron. J. Combin., 26 (4) (2019) \#P4.49.

[3] M. Axenovich, C. Winter. A note on saturation for Berge- $G$ hypergraphs. Graphs Combin., 35 (4): 933-939, 2019.

[4] S. English, P. Gordon, N. Graber, et. al. Saturation of Berge hypergraphs. Discrete Math., 342: 1738-1761, 2019.

[5] S. English, D. Gerbner, A. Methuku, M. Tait. Linearity of saturation for Berge hypergraphs. European J. Combin., 78: 205-213, 2019.

[6] P. Erdős, A. Hajnal, J. Moon. A problem in graph theory. Amer. Math. Monthly, 71: 1107-1110, 1964.

[7] J. Faudree, R. Faudree, J. Schmitt. A survey of minimum saturated graphs. Electron. J. Combin., 1000: 19-29, 2011.

[8] D. Gerbner, C. Palmer. Extremal results for Berge hypergraphs. SIAM J. Discrete Math., 31 (4): 2314-2327, 2017. 
[9] L. Kászonyi, Zs. Tuza. Saturated graphs with minimal number of edges. J. Graph Theory, 10 (2): 203-210, 1986.

[10] D. Korándi, B. Sudakov. Saturation in random graphs. Random Structures Algorithms, 51 (1): 169-181, 2017.

[11] A. Mohammadian, B. Tayfeh-Rezaie. Star saturation number of random graphs. Discrete Math., 341: 1166-1170, 2018.

[12] O. Parczyk, Y. Person. Spanning structures and universality in sparse hypergraphs. Random Structures Algorithms, 49 (4): 819-844, 2016.

[13] N. Salia, C. Tompkins, Z. Wang, O. Zamora. Ramsey numbers of Berge-hypergraphs and related structures. Acta Math. Univ. Comenianae, 88 (3): 1035-1042, 2018.

[14] M. Zito. Small maximal matchings in random graphs. Theoret. Comput. Sci., 297: 487-507, 2003. 